\title{
AMÉLIE GREGÓRIO, «L'Arabe» colonisé dans le théâtre français. De la conquête de l'Algérie aux grandes expositions coloniales (1830-1931)
}

\section{Elena Fermi}

\section{OpenEdition}

Journals

\section{Édition électronique}

URL : https://journals.openedition.org/studifrancesi/44413

DOI : 10.4000/studifrancesi.44413

ISSN : 2421-5856

Éditeur

Rosenberg \& Sellier

\section{Édition imprimée}

Date de publication : 1 juin 2021

Pagination : 253-254

ISSN : 0039-2944

\section{Référence électronique}

Elena Fermi, « AMÉLIE GREGóRIO, «L'Arabe» colonisé dans le théâtre français. De la conquête de l'Algérie aux grandes expositions coloniales (1830-1937)», Studi Francesi [En ligne], 193 (LXV | I) | 2021, mis en ligne le 01 juillet 2021, consulté le 15 octobre 2022. URL : http://journals.openedition.org/studifrancesi/44413 ; DOI : https://doi.org/10.4000/studifrancesi.44413

Ce document a été généré automatiquement le 15 octobre 2022.

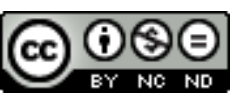

Creative Commons - Attribution - Pas d'Utilisation Commerciale - Pas de Modification 4.0 International - CC BY-NC-ND 4.0

https://creativecommons.org/licenses/by-nc-nd/4.0/ 


\title{
AMÉLIE GREGÓRIO, «L'Arabe» colonisé dans le théâtre français. De la conquête de l'Algérie aux grandes expositions coloniales (1830-1931)
}

\author{
Elena Fermi
}

\section{RÉFÉRENCE}

AMÉLIE GREGÓRIO, «L'Arabe» colonisé dans le théâtre français. De la conquête de l'Algérie aux grandes expositions coloniales (1830-1931), Lyon, Presses Universitaires de Lyon, 2020, 360 pp.

1 Dans sa thèse de doctorat, publiée aux Presses Universitaires de Lyon dans la collection «Théâtre et société», Amélie Gregório s’interroge sur le rôle de vecteur culturel joué par le théâtre populaire dans la diffusion, auprès du public français, de l'esprit colonial et sur sa contribution à l'imposition des idées de conquête et d'empire dans la société française $d u X x^{e}$ et du début $d u x^{e}$ siècle. L'auteure annonce, en introduction, les limites temporelles et géographiques à l'intérieur desquelles elle va conduire son étude. La période 1830-1931 identifie un siècle marqué à son début par la conquête de l'Algérie et à son terme par l'Exposition coloniale internationale «grand rendez-vous impérialiste [...], aboutissement de plusieurs événements de ce type, en France et dans le monde» (pp. 7-8) qui montrerait à quel point, après la Grande Guerre, l'imaginaire colonial semble fixé dans les esprits. Du point de vue géographique, la recherche se concentre sur la colonisation de l'Afrique du Nord, de l'Algérie en particulier, car l'auteure considère l'expansion coloniale au Maghreb comme «l'un des enjeux majeurs de la politique nationale dans la société française du $\mathrm{XIX}^{\mathrm{e}}$ et du début du $\mathrm{XX}^{\mathrm{e}}$ siècle» ( $\mathrm{p}$. 6). Le genre littéraire faisant l'objet d'analyse dans ce volume est le théâtre dans ses formes les plus populaires et le corpus pris en considération - extrêmement vaste et 
varié - va du vaudeville au drame, de la comédie romantique au spectacle équestre, de la tragédie au spectacle musical. L'étude est centrée sur la représentation de «l'Arabe», mot désignant au départ «celui qui vient d'Arabie» mais qui, au fil du temps, a pris un sens bien plus large, en identifiant l'ensemble des populations du sud du bassin méditerranéen. Pour mener à bien sa recherche, Gregório fouille dans les archives, redécouvre et analyse plus de 200 pièces (dont la plupart ne sont pas passées à la postérité). Elle en présente la trame, en examine la mise en scène et les décors, sans oublier d'offrir aux lecteurs un compte-rendu de leur réception de la part du public sur le continent et dans les colonies - mais également de la critique. Elle s'interroge ainsi sur la mesure exacte du rôle joué par le théâtre dans la politique d'expansion et de domination, sur la fréquence et les inflexions avec lesquelles il a joué ce rôle, sur les idées qu'il a diffusées, sur les différentes représentations de «l'Arabe» qu'il a véhiculées et sur sa prise de distance, voire sa contestation du phénomène colonial. La monographie, divisée en trois parties, présente une étude caractérisée par une perspective interdisciplinaire. Gregório fait se croiser histoire politique, culturelle et sociale pour mettre en lumière l'approche plurielle du théâtre dans ce questionnement. La première partie, "Un siècle de colonisation au prisme de l'art dramatique: les inflexions du discours théâtral sur "l'Arabe"”, présente un excursus qui, en confrontant histoire politique et histoire du théâtre, tente de montrer la manière dont la création dramatique accompagne dans le temps les faits coloniaux. Entre 1830 et 1850, une kyrielle de pièces militaires à grand spectacle et de vaudevilles anecdotiques accompagnent et célèbrent la conquête française dans une logique de célébration politique, conforme aux attentes de la censure. Le théatre se fait au cours de ces deux décennies la chambre d'écho de l'expansion coloniale. L'auteure se concentre donc sur les choix des auteurs dramatiques qui montreraient, selon elle, à quel point leur vision est partielle et manichéenne. La figure de l'indigène, «l'Arabe» - qu'il soit Arabe, Kabyle, Touareg ou métissé, car la distinction n'est pas de mise à l'époque - y paraît toujours amoindrie, stéréotypée, caricaturée face au Français. L'idée sous-tendue est celle de montrer la supériorité du colonisateur, d'en exalter la mission civilisatrice face à la sauvagerie des populations indigènes. De cette multitude d'Arabes privés de personnalité, Gregório fait émerger dans son étude celle de l'émir Abd-el-Kader, symbole de la résistance au colonisateur français en Algérie, dont la représentation évolue au fil du temps et des événements qui marquent sa vie, de la moquerie des premières pièces à une sorte de mythification qui frise parfois l'hagiographie dans les pièces plus tardives du XIX ${ }^{e}$ siècle, jusqu'à disparaître presque des œuvres qui marquent la scène au début du $\mathrm{xx}^{\mathrm{e}}$ siècle. Violence, traîtrise, mensonge et paresse caractérisent non seulement l'homme arabe mais également la femme, bien qu'elle soit bien moins représentée sur la scène. Le cliché féminin est cependant plus celui de la séductrice qui se sert de sa sensualité trouble et un peu animale pour séduire son entourage masculin et attiser la jalousie des rivales françaises. La représentation de «l'Arabe» ne cesse cependant d'évoluer et l'auteure montre comment on passe de l'exaltation de la bestialité indigène à la mise en scène d'une fraternité et d'une alliance possibles entre Français et indigènes qui caractérisent les pièces les plus récentes du corpus, dans lesquelles l'objectif reste pourtant celui de montrer les «bienfaits» de la domination.

2 La deuxième partie "Exotisme et altérité dans les mises en scène de l'autre et de l'ailleurs» se focalise sur la mise en scène de cet ailleurs qui est l'univers de «l'Arabe». C'est donc plus le fait théâtral qui est ici interrogé, du point de vue de la mise en scène, entendue comme l'ensemble des décors, des costumes, du maquillage, des 
chorégraphies, de la musique qui accompagnent les pièces, ainsi que des inflexions et des altérations arabisantes du langage qui y est employé. Gregório montre à quel point l'exotisme le plus conventionnel y est de mise, que ce soit dans les décors intérieurs où dominent harems et cafés maures ou dans les extérieurs où palmiers, désert et autres sites «sauvages» exaltent le pittoresque de l'atmosphère et contribuent à la construction d'un imaginaire qui accentue l'écart entre l'ici et l'ailleurs. Les costumes, le maquillage et la gestualité des comédiens participent eux aussi de cette fiction visuelle: qu'ils offrent une vision simplifiée et déformée ou qu'ils tentent d'être plus fidèles au réel, ils sont le miroir de leur temps et en révèlent la mentalité. Danse et musique arabes ou arabisantes ajoutent aux mises en scène une ultérieure touche d'exotisme, exaltée aussi par le langage. Clichés, stéréotypes et images attendues caractérisent les spectacles et leur réception publique mais les représentations évoluent au fil du temps et deviennent aussi une occasion de renouvellement.

3 La troisième partie "De la possibilité d'un autre discours dramatique sur "l'Arabe"”, est programmatique dès le titre. Gregório annonce vouloir enquêter les tentatives de dépassement des stéréotypes, à travers une étude renouvelée d'une partie du corpus déjà présentée et l'introduction d'œuvres qui n'avaient pas été précédemment abordées. L'accent va être mis sur l'émergence progressive d'un débat intellectuel autour du phénomène colonial qui, bien que souvent contradictoire, nuancé, tendu, va influencer les représentations théâtrales déjà au cours du XIX ${ }^{e}$ siècle, pour prendre de plus en plus d'ampleur au cours du $\mathrm{xx}^{\mathrm{e}}$. Des figures de l'entre-deux, remettant en cause l'opposition entre l'ici et l'ailleurs, commencent à apparaître dans les pièces et vont ouvrir le chemin à la pensée anticolonialiste. Un focus particulier est consacré dans cette partie à Henri-René Lenormand, pour la position très singulière qu'il tiendrait, selon l'auteure, dans la production théâtrale relative à l'Afrique du Nord colonisée au cours de la période faisant l'objet de l'analyse. Auteur à la fois de textes dramatiques et critiques, Lenormand interroge les rapports de domination, en faisant entrevoir la possibilité d'une remise en question qui n'aboutira que plus tard aux prises de position nettement anticolonialistes d'un Césaire ou d'un Genet.

Cette étude, très riche et très approfondie, se termine sur une question: «un tel patrimoine théâtral et culturel, à certains égards encombrant dans sa dimension idéologique, ne mériterait-il pas d'être redécouvert, reconsidéré sinon réhabilité, moins pour les réponses historiquement datées qu'il apporte sur le sujet de l'altérité que pour les questions qu'il continue de poser à notre société?» (p. 333). Le théâtre comme miroir d'une société, lieu de construction d'une pensée collective et partagée est ici mis à l'honneur. Il permet non seulement d'analyser le passé mais aussi de questionner le présent. La bibliographie annexe au volume révèle une grande richesse de documentation et la division des sources consultées selon les sujets qu'elles traitent permet de se repérer facilement. 